\title{
Study on the Frequency and Voltage Coordinated Control for Wind and Energy Storage System
}

\author{
Shanying $\mathrm{Li}^{1}$,Tao $\mathrm{Wu}^{1}$,Haiying Xing ${ }^{1}$, Tingjian $\mathrm{Han}^{1}$, Tianzhi Cao ${ }^{1}$, Xisheng \\ Tang $^{2}$ and Chao Gao ${ }^{2}$ \\ ${ }^{1}$ NO.1.Dizang'an Nanxiang.FuxingmenwaiDajie,Beijing.P.R.China \\ ${ }^{2}$ No. 6 Zhongguancun, HaidianDistric, Beijing.P.R.China \\ *lee22yanjin@126.com
}

\begin{abstract}
Keywords: The frequency and voltage coordinated control, wind/storage system, real time digital simulation (RTDS).

Abstract: Due to the characteristics of the operation of the wind turbine and the capacity of the converter and the rotor current and other factors, the use of wind turbine to adjust the system frequency and voltage can not fully meet the requirements. And energy storage equipment has the advantages of flexible and controllable. In the paper, the frequency and voltage coordinated control for wind power and energy storage system is studied. And its coordinated control strategy is proposed. Three kinds of control scheme comparison are considered in the simulation to verify the validity of the control strategy proposed. The simulation results show that the control strategy is effective and easy to apply engineering practice.
\end{abstract}

\section{Introduction}

With the advance of large-scale wind power integration, the total amount of power generation increases. At this time, if the wind turbine is not involved in the system frequency regulation, the equivalent power generation capacity and the inertia of the rotor are reduced, which causes the faster reduce or increase the system frequency[1]. In addition, the frequency of the system may also be difficult to recover to normal levels after the disturbance disappeared [2-3]. At present, the double fed wind induction generator (DFIG) is used for system frequency modulation. Compared with the conventional unit to participate in the system frequency regulation system, the DFIG is generally operated under the maximum power point tracking (MPPT) mode. So when the frequency of the system is reduced, it is unable to provide the additional power to adjust the frequency. The energy storage device has the advantages of fast response, precise control, bidirectional adjustment, flexible and controllable. When the wind speed is too low (close to cut in wind speed), the frequency regulation of the wind turbine is limited, we can use the energy storage equipment with the wind turbine as the frequency regulation standby

If the wind farm is equipped with the appropriate capacity of the energy storage equipment, which plays a fast charge and discharge of the storage device, flexible control of reactive power, it can effectively restrain the voltage fluctuation caused by wind power integration, and improve the voltage stability of the power system[4-6]. The system frequency and voltage regulation with wind energy storage cluster can make full use of the advantages of fast speed control of the wind turbine, the control of the variable pitch control, the wide adjustment range and the stability of the energy storage device. And it can compromise the factors that the limit of the wind turbine speed, the response time of the variable pitch control, the frequent participation in the adjustment of the service life, and the high cost of energy storage equipment.

So the frequency and voltage coordinated control strategy for wind power and energy storage system is proposed in the paper. And it is designed with the real time digital simula ${ }^{1}$ tion (RTDS) platform. The capacity limit of the wind turbine and the energy storage are considered in the proposed method, according to the operation mode of the electric power system, the coordination control is realized. The frequency regulation scheme is achieved based on the use of the wind turbine speed 
control, variable pitch control combined and the energy storage device. The voltage regulation scheme is realized by using the rotor current excitation component and the grid side converter of the wind turbine. The coordinated control strategy can improve the overall economic benefits of the wind/energy cluster operation, at the same time to meet the needs of the system frequency and voltage regulation.

\section{Frequency and Voltage Coordinated Control Strategy for Wind Power and Energy Storage System}

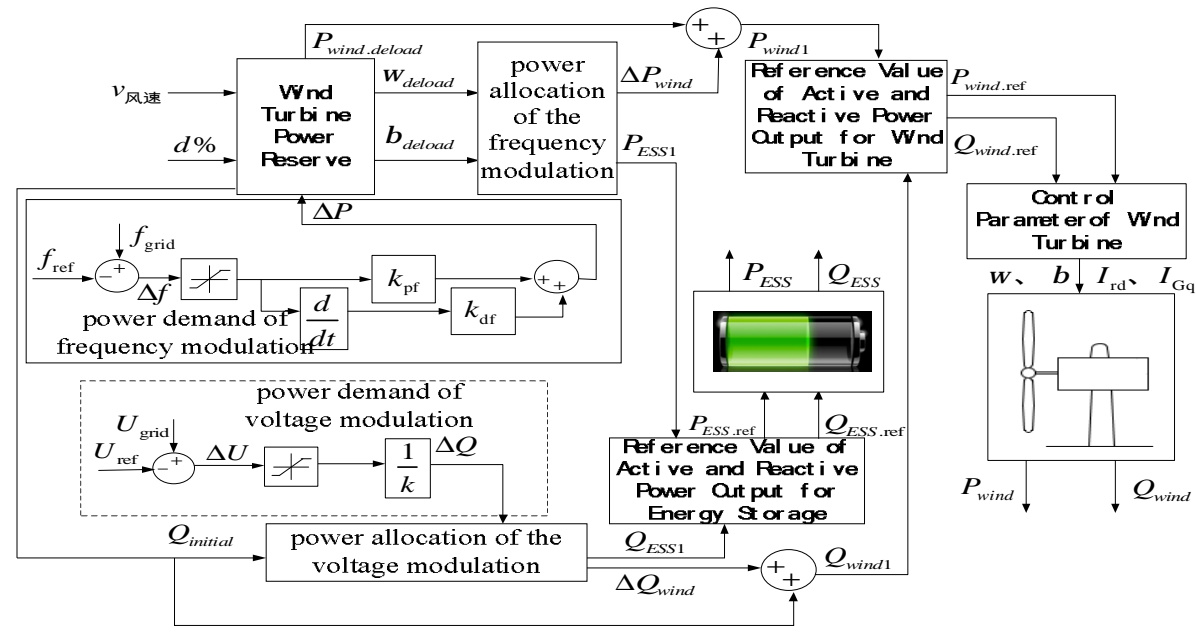

Fig.1 Frequency and VoltageCoordinated Control Strategyfor Wind/Storage System

The frequency and voltage coordinated control strategy for wind/storage system is shown in Fig.1.The power system frequency and voltage regulation can be divided into three control modules by using wind power and energy storage cluster. They are frequency modulation module, voltage modulation module and frequency and voltage coordinated control module. The frequency modulation module includes the wind turbine power reserve, the power allocation of the frequency modulation, the power demand of frequency modulation. The voltage modulation module includes the wind turbine power reserve, the power allocation of the voltage modulation, the power demand of voltage modulation. The frequency and voltage coordinated control module includes reference value of active and reactive power output for wind turbine and energy storage.

According to the magnitude of the system frequency and voltage change, it can be divided into 16 kinds of working conditions, and then it can be concluded as 3 kinds of working conditions, which can realize the coordinated control of the frequency and voltage regulation (Fig.2).

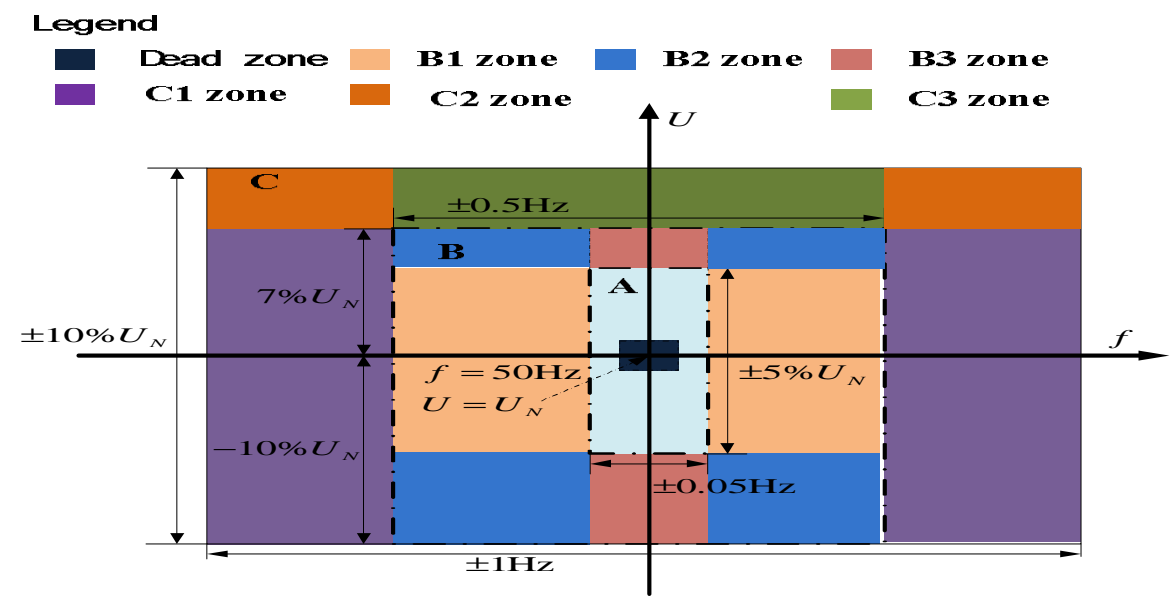

Fig.2 Electrical state partition diagram 
According to the regional distribution in Fig.2, when the electrical state of the detection to the power system in the area $\mathrm{B} 1$ and $\mathrm{C} 1$ zone, the system frequency regulation demand more obvious. At this point, we should give priority to ensuring the power system frequency requirement. At this time, wind storage cluster for capacity allocation mainly to meet the demand of active power rate, namely coordination control strategy in frequency priority mode, in case 1 representation (Fig.3). When the electrical state is in area A, B3 and C3 zone, the system voltage demand is more obvious. At this point, you should give priority to ensuring the power system voltage requirements. At this time, wind storage cluster in capacity allocation mainly to meet the reactive power demand, the coordination control strategy in voltage priority mode, expressed in case3 (Fig.3). When in the electrical state of the detected power system outside the B2, C2 and C district, voltage and frequency regulation demand is the same, without focus. At this point, you should also guarantee power system frequency and voltage requirements, at this time, wind storage cluster in capacity allocation of reactive power and reactive power distribution of the same priority, in meet the wind cluster storage capacity requirements, system vacancy in proportion to the allocation of active and reactive power according to the, namely, in no priority mode, expressed in case 2 (Fig.3).In the same way, reference value of active and reactive power output for energy storage is shown in figure 4.

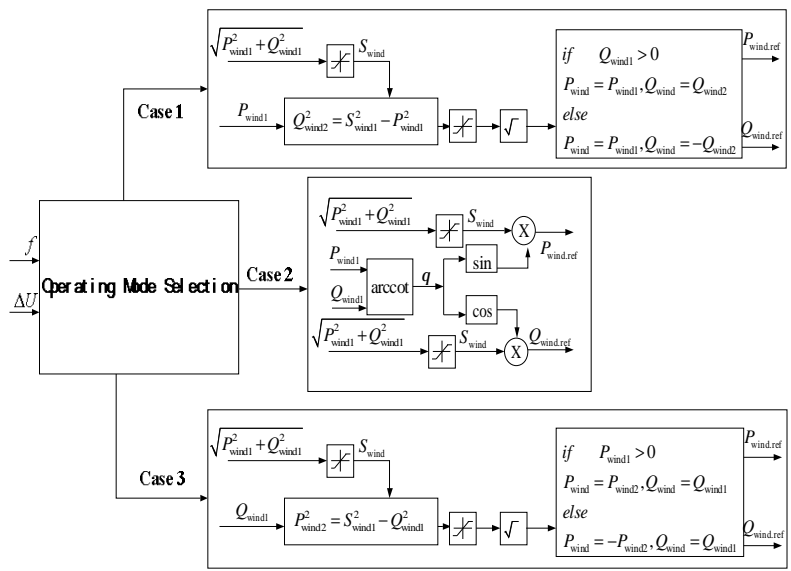

Fig.3 Reference Value of Active and Reactive Power Output for Wind Turbine

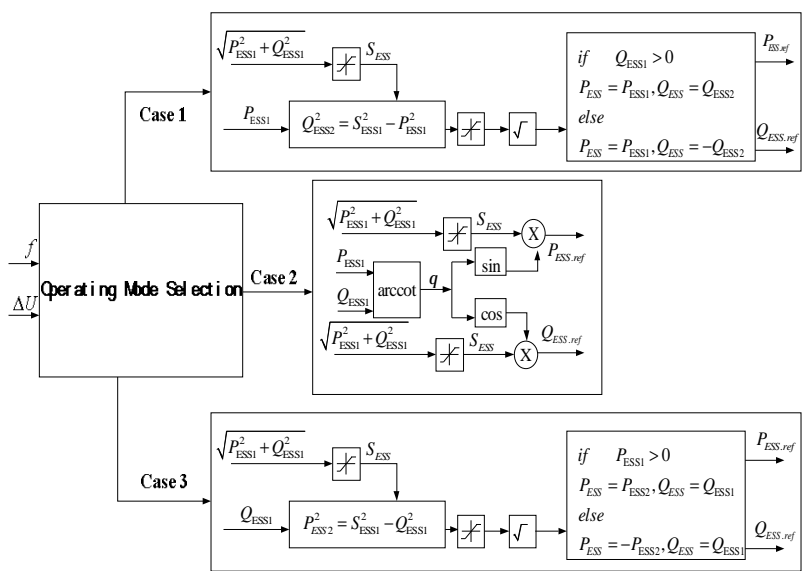

Fig.4 Reference Value of Active and Reactive Power Output for Energy Storage

\section{Test of Frequency and VoltageControl of Wind Power and Energy Storage System}

\section{Test System}

In order to verify the coordinated control strategy proposed in the section 2 , the simulation is carried out RTDS experimental platform. The test system is typical three generators and nine buses system in western United States. Considering the wind turbines and energy storage to load node is the most serious impact on the system, they are installed in bus 8 . The rated capacity of wind turbine is 2.2 MW, installing 70 units (shown in figure 5).

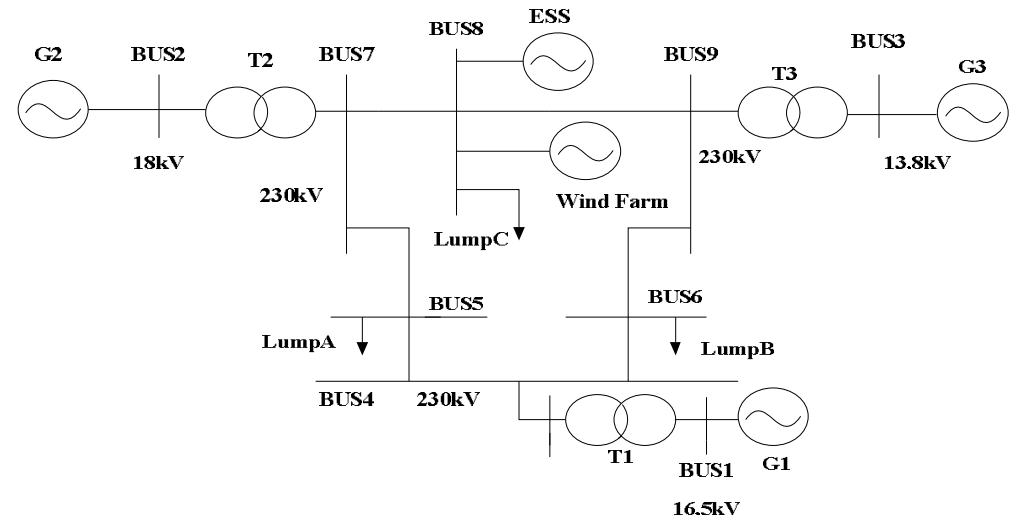

Fig.5 Test System Diagram 


\section{Test of Frequency Control for Wind Power and Energy Storage System}

To test the effect of the system frequency regulation of control scheme with wind/storage cluster, simulation shows the three kinds of scheme comparison (Fig.6), case 1 for wind turbine and storage are not involved in the frequency control (black curve), only wind turbine involved for case 2 (red curve), case 3 for the control scheme proposed in the paper (blue curve). In the simulation, the wind speed is $9 \mathrm{~m} / \mathrm{s}$, the disturbance is the active load increase $30 \mathrm{MW}$ at bus 8 .

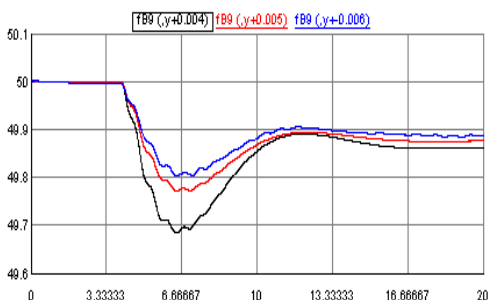

(a) System Frequency

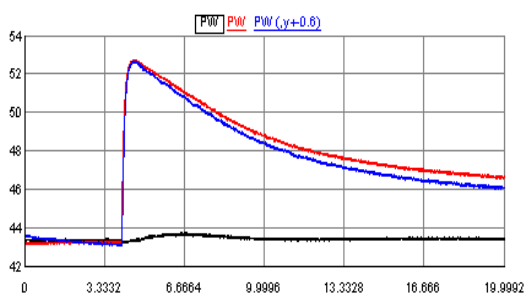

(b) Wind Turbine Active Power

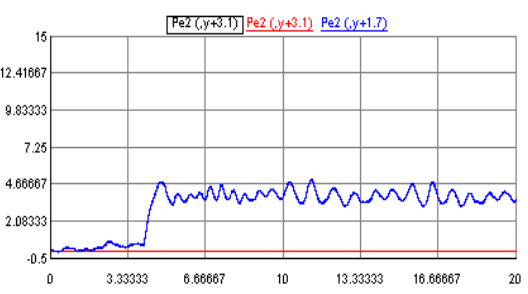

(c) Energy Storage Active Power

Fig. 6 the Results of the Simulation (wind speed 9m/s, active load increase 30MW at Bus 8 )

The simulation results shows, if the system frequency detected declines, it needs certain active power frequency modulation. When wind turbine and storage are not involved in frequency, the frequency quality of the system can meet grade B. When wind turbine regulation strategy is adopted, the maximum transient frequency offset is $0.22 \mathrm{~Hz}$, the steady state frequency offset is $0.13 \mathrm{~Hz}$. In wind and storage both involved in frequency control strategy, the maximum frequency deviation is $0.19 \mathrm{~Hz}$, the steady-state deviation is $0.12 \mathrm{~Hz}$.Thus, the frequency regulation scheme proposed in section 2 has better effect of frequency modulation.

\section{Test of Voltage Control of Wind Power and Energy Storage System}

In order to verify the effect of the voltage regulation of the proposed scheme with wind/storage cluster. Simulation shows the three kinds of scheme comparison(Fig.7), case 1 for wind turbine and storage are not involved in the voltage control (black curve), only wind turbine involved for case 2 (red curve), case 3 for the control scheme proposed in the paper (blue curve).In the simulation, the wind speed is $9 \mathrm{~m} / \mathrm{s}$, the disturbance is the reactive load increase $50 \mathrm{MW}$ at bus 8 .

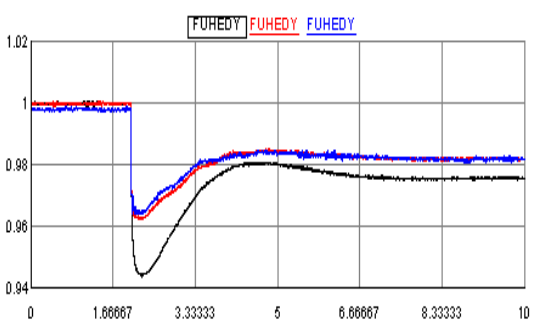

(a) System Frequency

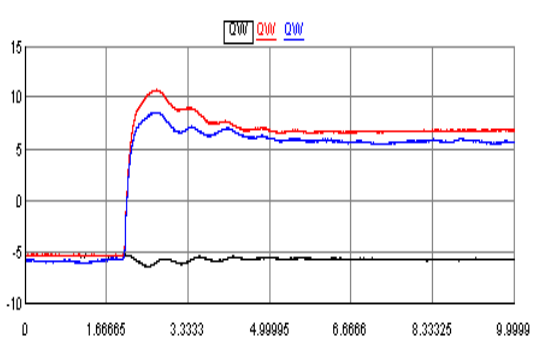

(b) Wind Turbine Reactive Power

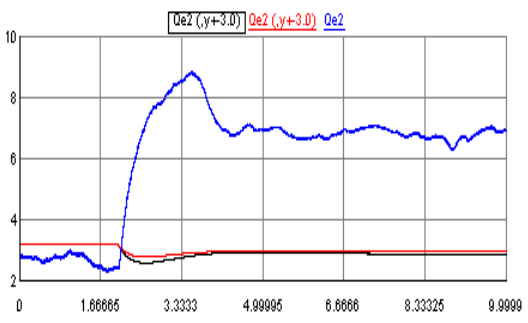

(c) Energy Storage Reactive Power

Fig. 7 the Results of the Simulation (wind speed $9 \mathrm{~m} / \mathrm{s}$, reactive load increase $50 \mathrm{MW}$ at bus 8 )

If the system voltage drops, the need to increase the reactive power to adjust the voltage. When the wind speed is low, the wind turbine can adjust the reactive power by the rotor excitation and the grid side converter. When there is reactive power fluctuations, the system voltage regulation can be carried out by the wind turbine and energy storage cluster. In the regulation process, if the wind turbine is not involved in the voltage regulation of the power system, the voltage quality will be reduced to the $\mathrm{B}$ level in the transient process. While the wind/storage cluster are involved in the voltage regulation, even in the transient process, the voltage quality is also a grade A. It can be seen that the voltage regulation scheme with wind/storage cluster has good effect on voltage regulation.

\section{Test of Frequency and Voltage Coordinated Control of Wind Power and Energy Storage System}

Simulation and verification are carried out for single phase grounding fault in bus 9, the results are shown in figure 8. Single phase grounding fault occurred in bus 9. Simulation shows the three kinds of scheme comparison, case 1 for wind turbine and storage are not involved in the frequency and voltage 
coordinated control (black curve), only wind turbine involved for case 2 (red curve), case 3 for the control scheme proposed in the paper (blue curve).

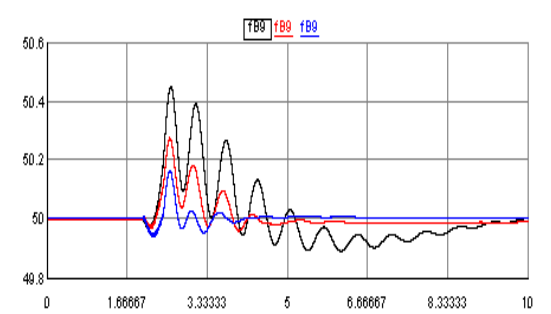

( a ) the system frequency

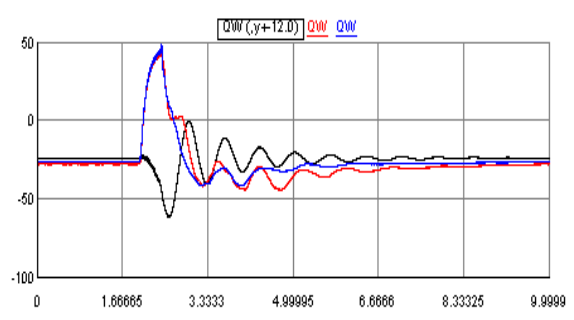

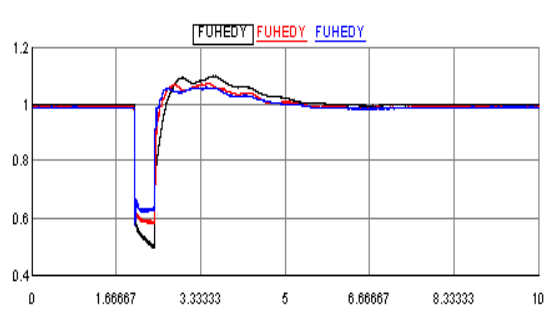

( b ) the system voltage

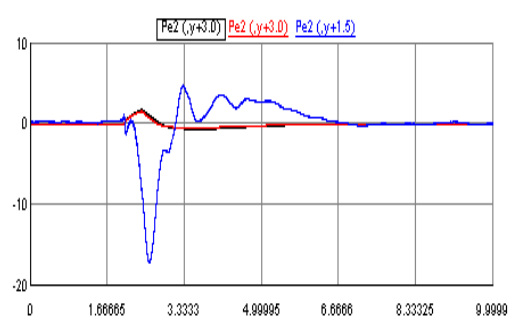

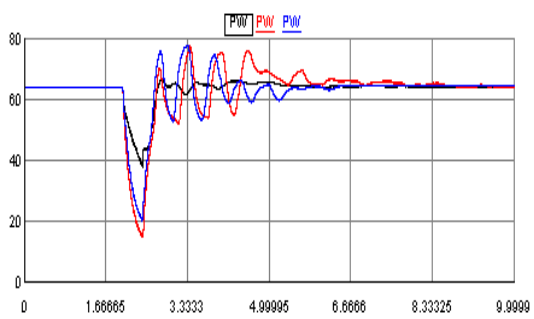

(c ) the wind turbine active power

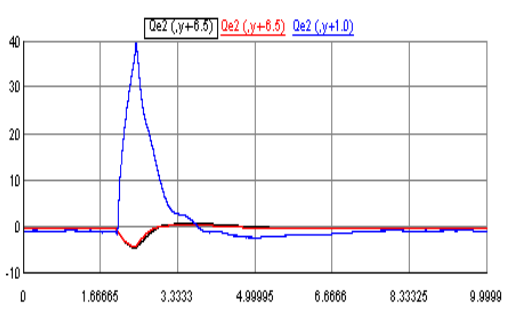

( d )Wind Turbine Rective Power( e )Active Power of the Energy Storage( f )Reactive Power of the Energy Storage

Fig. 8 the Results of the Simulation (wind speed $10 \mathrm{~m} / \mathrm{s}$, Single phase grounding fault at bus 9 )

The simulation results shows that when the single phase grounding fault ocurred, the voltage adjustment demand is more urgent than the frequency adjustment. Thus the system voltage demand is under the priority consideration for the coordinated control. So the coordinated control of the wind energy storage cluster is a good way to improve the system's failure through the issuance of reactive power. In the short circuit, the coordinated control strategy of wind/storage cluster can be used to suppress the frequency oscillation, and also have good effect on the frequency adjustment.

\section{Conclusion}

Based on the RTDS platform, a hybrid simulation system and a full digital real time simulation system of the DFIG and energy storage system are built. The scheme of the frequency and voltage regulation for the wind turbine and energy storage system is discussed in the paper. The simulation is carried out with the three kinds of frequency and voltage control scheme comparison. The results show that the control scheme proposed in the paper is feasible and effective.

\section{References}

[1] N. W. Miller, Frequency responsive wind plant controls: impacts on grid performance. IEEE in Power and Energy Society General Meeting, San Diego, CA, USA, 2011, pp.1-8

[2] J.Aho, A. Buckspan, Tutorial of wind turbine control for supporting grid frequency through active power control. American Control Conference, Montreal. Canada, 2012, pp. 3120-3131.

[3] Q.Jiang, H.hong, Wavelet-based capacity configuration and coordinated control of hybrid energy storage system for smoothing out wind power fluctuation. IEEE Trans on Power Systems, Vol.28, no.2, 2013, pp.1363-1372.

[4] Xisheng Tang,etal, Wind farm active power control based on energy storage system . IWEC 2014, pp.1-6.

[5] Meghdad Fazeli, Greg M. Asher, etc., in novel integration of wind generator-energy storage systems within microgrids. IEEE Trans. on Smart Grid, Vol.3, no.2, 2012, pp:728-738 
[6] Makarov Y.V., Pengwei Du, etc., in sizing energy storage to accommodate high penetration of variable energy resources. IEEE Trans. on Sustainable Energy, Vol.3, no.2, 2012, pp: 34-40 